\title{
Shape effects in biological adhesion of ellipsoidal cells
}

\author{
Mikhail Kaznacheev ${ }^{1, *}$ and Aleksey Belyaev ${ }^{2,3, * *}$ \\ ${ }^{1}$ Department of Mathematics, Faculty of Physics, M.V.Lomonosov Moscow State University, Moscow \\ 119991, Russia \\ ${ }^{2}$ Department of Biophysics, Faculty of Physics, M.V.Lomonosov Moscow State University, Moscow \\ 119991, Russia \\ ${ }^{3}$ Interdisciplinary Research Center Mathematical Modelling in Biomedicine, RUDN University, 117198 \\ Moscow, Russia
}

\begin{abstract}
Blood platelet adhesion is crucial for arterial thrombosis and hemostasis. The attachment of platelets to the injuries takes place under the action of high hydrodynamic forces and relies on the formation of breakable ligand-receptor bonds between the cell and the adhesive substrate. In this work we study how the geometrical effects may change the adhesive forces that stick platelets to the wounds. The mathematical model shows that oblate cells with high aspect ratio are more favourable for thrombus growth.
\end{abstract}

\section{Introduction}

Blood platelets play a key role in arterial and microvascular thrombosis, where the intensive hemodynamic flows and near-wall viscous stresses hinder the clotting of blood plasma [1, 2]. Physiological functions of platelets rely on their ability to adhere to a wound (or to a thrombus) and to withstand relatively mechanical stresses in the flowing blood [3]. In biological systems the adhesion mechanisms are mainly based on key-lock interactions of special protein molecules on the outer side their membranes with different adhesive substrates (e.g. collagen fibers, von Willebrand factor and membrane receptors of other cells) [1, 57]. The ligand-receptor bonds thus represent elastic bridges that emerge and dissociate with different kinetics, depending on the mechanical actuation and the molecular interactions of the involved proteins $[8,9]$. A number of experimental techniques has been used to study the adhesive forces between platelets, most of which rely on mechanical separation of the adhering surfaces [10-12]. We concentrated our mind on geometric aspect of the problem. E.g. in work [13] authors investigated the influence of the form on collision effects, this required quite hard computational model. We tried to create a model which gives quantitative results in easier way. In this study, we hypothesised that the shape of the cell may cause a significant alteration of adhesive forces. We developed a mathematical model for the ligandreceptor adhesion of a blood platelet with a solid wall, covered by adhesive ligands. The model shows that the aspect ratio of the platelet is an important parameter, and that oblate cells are able to withstand greater pulling forces.

\footnotetext{
*e-mail: kaznacheev.michael@mail.ru

**e-mail: aleksey_belyaev@yahoo.com
} 


\section{Materials and Methods}

\subsection{Mathematical Model}
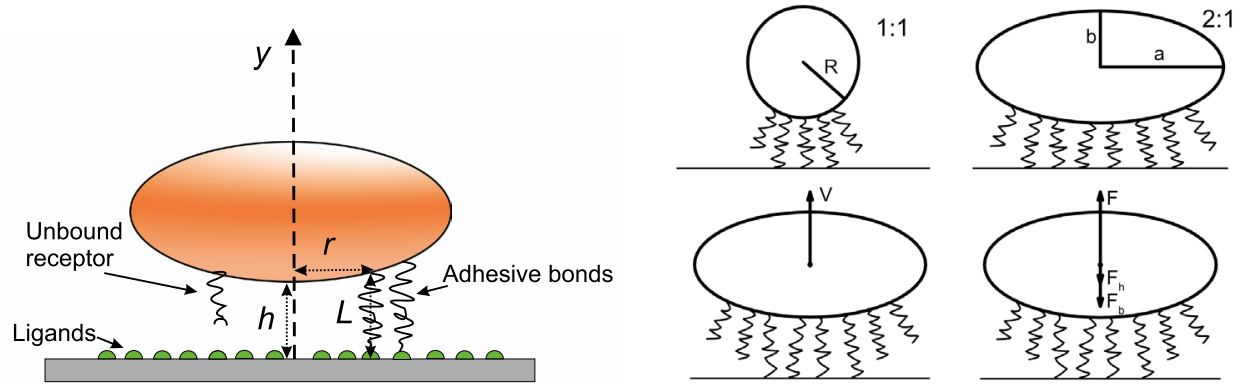

Figure 1. The scheme of the studied problem (left) and the illustration of the considered cases (right). The dynamics of adhesion of oblate ellipsoidal cell is considered for cells of different aspect ratios. The constant-velocity and constant-force regimes were studied.

We consider the biological adhesion of a platelet to a flat surface $y=0$ evenly covered with adhesive ligand molecules (Fig. 1). We assume that the platelet surface is also uniformly covered with adhesion receptors. In addition, we may assume that only vertical bonds are formed. Then, the problem can be treated in a cylindrical system of coordinates $(r, \varphi, y)$. In our model we consider the adhesion bonds to be springs with the equilibrium length $\lambda$ and elasticity constant $\kappa_{0}$. The probability of bond formation is determined by the constants of forward and backward reaction rates between adhesion receptors and ligands. The platelet is approximated in this model by a rigid nondeformable ellipsoid of revolution. This is an admissible approximation, because in the deactivated state the platelet hardness is provided by the cytoskeleton [3], the platelet Young's modulus in this state is approximately $170 \mathrm{pN}$. $\mu m^{-2}$ [4]. Considering values of forces below it is sufficient to use such simplification. We consider the situation where the platelet moves only in vertical direction (normally to the wall). Different aspect ratios of the cell were considered in the presented study (Fig. 1).

The dynamics of the system relies on the reversible process of formation and dissociation of the adhesive bridges (or bonds) between the membrane receptors of the cell and the adhesive substrate (presented by immobilized protein ligands, e.g. collagen or von Willebrand factor [1]) on the wall. Since the number of adhesive receptors on the cell is typically very high $\left(10^{4}-10^{5}\right)$, we describe this process in terms of bond density $n(r, t)$. The latter evolves in time according to the kinetic equation $[8,9]$ :

$$
\begin{array}{r}
\frac{d n(r, t)}{d t}=K_{\text {on }}(r, t)\left(N_{r}-n\right)\left(N_{l}-n\right)-K_{\text {off }}(r, t) n(r, t), \\
K_{\text {on }}(r, t)=K_{\text {on }}^{0} \exp \left[\frac{-\kappa_{s}(L(r, t)-\lambda)^{2}}{2 k_{B} T}\right], \\
K_{\text {off }}(r, t)=K_{\text {off }}^{0} \exp \left[\frac{\left(\kappa_{0}-\kappa_{s}\right)(L(r, t)-\lambda)^{2}}{2 k_{B} T}\right],
\end{array}
$$

where $N_{r}$ is the density of receptors, $N_{l}$ is the density of ligands, $K_{\text {off }}^{0}$ and $K_{\text {on }}^{0}$ are the constants of reaction rates in the equilibrium state, $L(r, t)$ is the bond length, $k_{B}$ is the Boltzmann constant, $T$ is the temperature, $\kappa_{0}$ and $\kappa_{s}$ are the spring constants, and the sign in the second 
exponent determines the type of the bonds. Due to the axial symmetry of the system, there is no dependence on the azimuth angle $\varphi$ in the equation. The bond length is given by

$$
L(r, t)=h(t)-b\left(1-\frac{r^{2}}{a^{2}}\right)^{1 / 2}+b,
$$

where $a$ and $b$ are the ellipsoid semi-axes, $h$ is the shortest distance between the ellipsoid and the surface at the initial time, $r$ is the distance from the center of the system to the considered point, and $t$ is the time.

The difference between different types of ligands (or/and receptors) can be taken into account by using different constants of reaction (i.d. different $K_{o f f}^{0}, K_{o n}^{0}$ ). Below in Fig. 2(a) we represent the force kinetic with different value of $K_{e q}=\frac{K_{o n}^{0}}{K_{o f f}^{0}} N_{l}$. This means changing the $K_{\text {on }}^{0}$ or $K_{\text {off }}^{0}$ (more in sec 3.1).

The force acting upon the platelet is computed also by integrating over all possible positions of bonds with account for the tension of bonds. Because we consider only vertical bonds, the force has the only component in the $y$-direction given by:

$$
F_{b}(t)=\kappa_{0} \int_{0}^{a} \int_{0}^{2 \pi} n(r, t)[L(r, t)-\lambda] r d r d \varphi=2 \pi \kappa_{0} \int_{0}^{a} n(r, t)[L(r, t)-\lambda] r d r .
$$

In our model we investigate only vertical bonds, but on $2 \mathrm{D}$ case, thus we go to the polar coordinate system. This leads to necessity to integrate over a circle thus we have the factor $r$ and integral over $d r d \varphi$ in spite of nothing depends of $\varphi$. So, it gives the factor $2 \pi$ in the final result. Hence in reality we have the 1D integral over $r$ only.

In this study we modelled two different situations: pulling the platelet from the wall (i) with a constant vertical velocity $V$, and (ii) by a constant external force $F$ directed vertically, Fig.1. These are typical setups for measuring interfacial forces by Surface Force Apparatus [12]. For the constant velocity regime we measured the adhesive force $F_{b}$, and the distance between the platelet and the wall was explicitly time-dependent:

$$
h(t)=h_{0}+V \cdot t
$$

In the case of pulling by the externally applied force $F$, we also should take into account the viscous hydrodynamic forces arising from the fluid motion around the platelet. Naturally, the Reynolds number for a platelet-sized particle in a fluid with the viscosity of blood plasma is of order of 0.1 and less. Therefore, in our model the hydrodynamic interaction is characterized by a lubrication force $[12,14]$, given by the following formula

$$
F_{h}=\frac{6 \pi \eta R_{\mathrm{eff}}^{2} \frac{d y}{d t}}{y}, \quad R_{\mathrm{eff}}=\frac{a^{2}}{b},
$$

where $R_{\mathrm{eff}}$ is the effective hydrodynamic radius, $\eta$ is the dynamic viscosity, $y$ is the distance between the cell membrane and the wall. On assuming the over-dumped dynamics of the cell at low Reynolds numbers, the equation for the motion of the platelet may be derived from the previous expression:

$$
\frac{d y(t)}{d t}=\frac{1}{6 \pi \eta R_{\mathrm{eff}}^{2}}\left[F-F_{b}(t)\right] \cdot y(t),
$$

where $F$ is the external (pulling) force on the platelet and $F_{b}$ is the tension force of adhesive bonds. The latter one is obtained by a summation over all attached (or active) bonds at each 
moment of time. Thus, we solve Eq.(1) for the bond density at a given moment of time, compute the force of adhesion interaction with formula (5), and then use Eq. (8) to find the platelet position in the next moment time.

Solving the kinetic equation can give quantitative information about influence of the certain aspect ratio on the adhesion process. Moreover, the influence of other parameters like receptor density, bond constants, force or velocity can be investigated. The kinetics of the adhesion process may also be derived from the mathematical model.

Table 1. Model parameters

\begin{tabular}{|c|c|c|c|}
\hline Parameter & Symbol & Value & Dimension \\
\hline Initial distance & $h_{0}$ & 0.1 & $\mu \mathrm{m}$ \\
\hline Length of relaxed bond & $\lambda_{0}$ & 0.1 & $\mu \mathrm{m}$ \\
\hline Bond formation rate & $K_{\mathrm{on}}^{0}$ & $10^{-15}$ & $\mu \mathrm{m}^{2} \cdot \mathrm{s}^{-1}$ \\
\hline Bond dissociation rate & $K_{\mathrm{off}}^{0}$ & 10 & $\mathrm{~s}^{-1}$ \\
\hline Spring constant & $\kappa_{0}$ & $10^{-5}$ & $\mathrm{~N} \cdot \mathrm{m}^{-1}$ \\
\hline Spring constant & $\kappa_{s}$ & $0.5 \cdot 10^{-5}$ & $\mathrm{~N} \cdot \mathrm{m}^{-1}$ \\
\hline Thermal energy & $k_{b} T$ & $4,14 \cdot 10^{-21}$ & $\mathrm{~J}$ \\
\hline Density of receptors & $N_{r}$ & $10^{4}$ & $\mu \mathrm{m}^{-2}$ \\
\hline Density of ligands & $N_{l}$ & $10^{4}$ & $\mu \mathrm{m}^{-2}$ \\
\hline
\end{tabular}

\subsection{Numerical Methods}

To solve the differential equation we use the one stage Rosenbrock scheme with a complex coefficient [15]. We controlled the accuracy using the recurrent Richardson method. We consider the Cauchy problem

$$
y^{\prime}=f(y, t), \quad y(0)=y_{0},
$$

where $y$ is the sought function and $f(y, t)$ is the given function. For this problem, the CROS1 scheme has the form

$$
\begin{gathered}
{\left[1-\tau \alpha f_{y}\left(y_{n}, t_{n}\right)\right] w_{n}=f\left(y_{n}, t_{n}+\frac{\tau}{2}\right),} \\
y_{n+1}=y_{n}+\tau \operatorname{Re}\left(w_{n}\right),
\end{gathered}
$$

where $\alpha=\frac{1+i}{2}$, and $\tau$ is the time step. For each $r$ the bond kinetics Eq. (1) is an ordinary differential equation solvable with the numerical method Eqs. (10) and (11). We ensure the convergence of the numerical scheme by Richardson's procedure, which is based on mesh refinement [15]:

$$
R_{k N}=\frac{y_{r N}-y_{N}}{r^{p}-1}, \quad p_{k}=\frac{\lg \frac{R_{k-1}}{R_{k}}}{\lg r},
$$

where $k$ is the index that determines the number of the mesh ( 0 is the initial mesh, 1 is the mesh refined once, and so on), $r$ is the factor of mesh refinement (in what fold the mesh is refined in comparison with the previous one), $R_{k N}$ is an inaccuracy of the numerical solution, $y_{N}$ is the numerical solution to the equation, $y_{r N}$ is the solution on a refined mesh, and $p=2$ is the accuracy of the numerical scheme. Here $p_{k}$ represents the a posteriori accuracy of the numerical scheme, calculated according to Eq.(12) with several different meshes. If $p_{k}$ converges to the theoretical value $p=2$ upon increase of the mesh density, then the numerical scheme works correctly. 


\section{Results}

\subsection{Pulling with a constant velocity}

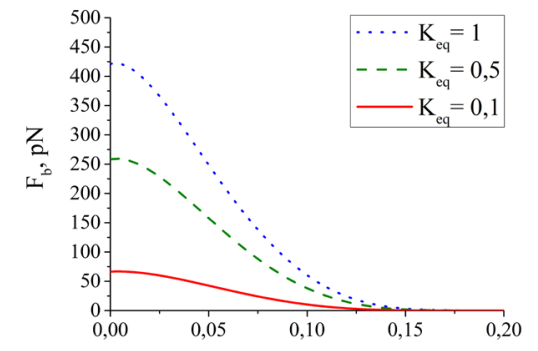

(a)

$\mathrm{t}, \mathrm{s}$

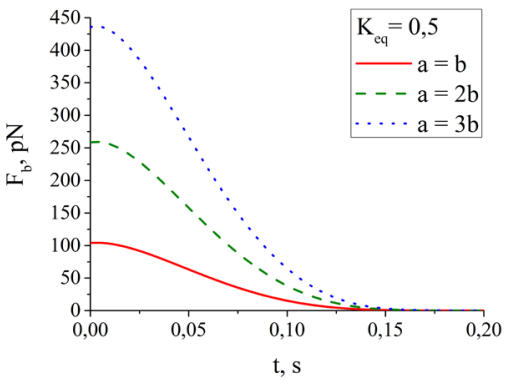

(b)

Figure 2. The adhesive force $F_{b}$ over time in the constant velocity case for different $K_{\text {on }}$ (left) and various aspect ratios of the platelet (right).

Firstly we investigated the dependence of the adhesive force on the binding kinetics (Fig. 2(a)). We altered the equilibrium constant for the binding-dissociation reactions $K_{\text {eq }}=K_{\text {on }}^{0} / K_{\text {off }}^{0}$. When $K_{\text {eq }}$ becomes smaller, the force $F_{b}$ decreases as well.

We studied the dependence of the simulation results on the shape of a platelet Fig.2(b). The semi-axes were chosen in the following manner. For the reference, we took the aspect ratio $2: 1(a=1.5 \mu \mathrm{m}$ and $b=0.75 \mu \mathrm{m})$ which is a typical value for human blood platelets. We altered the aspect ratio, keeping the platelet volume $\left(7.07 \mu \mathrm{m}^{3}\right)$ constant. The velocity of the platelet motion in these calculations was set to $0.4 \mu \mathrm{m} \cdot \mathrm{s}^{-1}$. As the ratio of semi-axes $a: b$ increases, the adhesive force also increases, which is a consequence of the larger area on which the bonds may be formed. Thus, for the aspect ratio $3: 1$, the force of adhesion appears to be more than twice that for $1: 1$.

Other way to increase the force is to increase the receptors density. Increasing the number of receptor density and the flattening a platelet can work together in increasing the ability of adhesion. We suppose that these mechanisms have important role both in vivo. The increase of the receptor density and the flattening of the platelet can work together enhancing the adhesion. But they have different impact on the number of bonds. Fig.3(a) shows how the number of bonds depends on the aspect ratio, here the density of receptors is $10^{4} \mu \mathrm{m}^{-2}$. The next picture (Fig.3(b)) represents how the kinetics depends on different numbers of receptors for a given aspect ratio $2: 1$. We can see that the number of bonds can significantly change if the receptor density is altered by the order of amplitude or more. The alteration of the aspect ratio also leads to a fast change of the amount of bonds. Therefore, flattening of the shape is a an efficient way to attain strong adhesion, especially when the receptor density is already very high and/or can not be increased easily. We also note that the aspect ratio governs the distribution of the bonds over the platelet membrane (Fig.3(c)) and, consequently, the distribution of the mechanical tension arising from the bonds.

\subsection{Pulling by a constant force}

The width of the minimum gap between the platelet and the plane has the form depicted in Fig.4 at the constant force $F=200 \mathrm{pN}$. At the semi-axes ratio $3: 1$ the larger number of bonds are generated than at the semi-axes ratio $1: 1$. Therefore, a spherical cell $(1: 1)$ is 
(a)

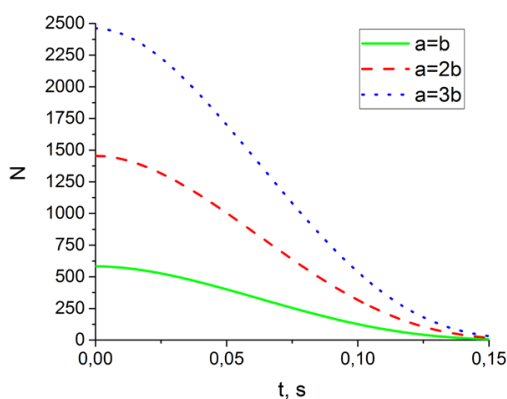

(b)

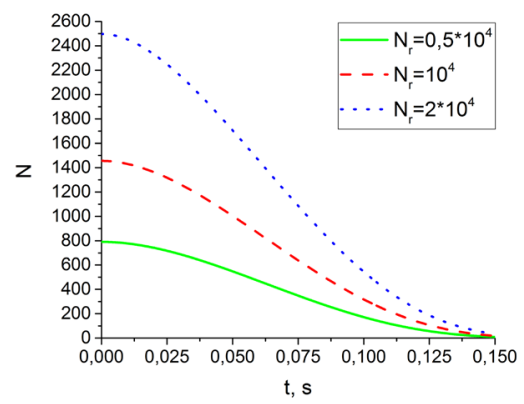

(c)

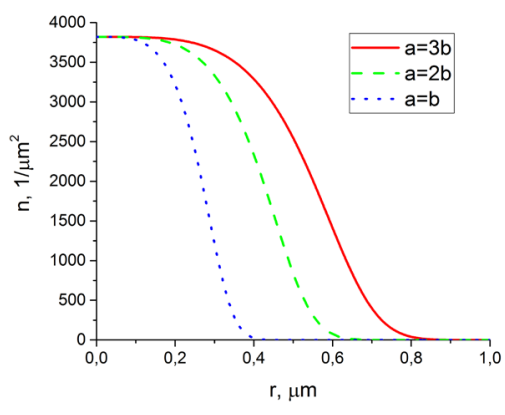

Figure 3. (a) Number of bonds $N$ over time in the constant velocity case for different aspect ratio. (b) Number of bonds $N$ over time in the constant velocity case for different receptor densities $N_{r}$. (c) Bond density $n$ over the distance from the center of the cell $r$.

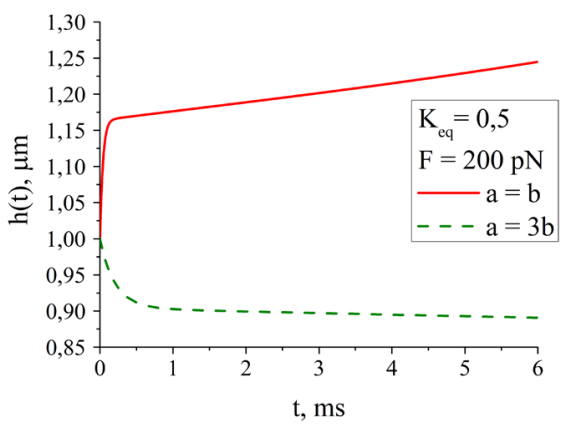

Figure 4. Width of the minimum gap between the platelet and plane over time, $\mathrm{F}=200 \mathrm{pN}$.

not able to form the required amount of bonds which gives sufficient value of force to stay the platelet near the wall. The spherical cell is detached from the surface, being pulled by the external force to the bulk of the fluid, Fig 4. As the semi-axes ratio increases, the shape of the cell becomes more plate-like, and the effective area of the platelet is larger and, as a consequence, a larger number of bonds is formed, hence if the $F_{b}$ is high enough, it balances the external force $F$, and the platelet rests on the wall, Fig 4. Shape is thus an important factor for platelet adhesiveness, which may have important implications for the mechanics 
and rheology of platelet thrombi. Oblate spheroidal shape favours the bond formation, so that platelets with high aspect ratio can withstand greater pulling forces.

\subsection{Discussion}

The results provide a numerical basis for the interpretation of measurements of biological adhesion forces, depending on the cell's shape, as well as the density of cell adhesive molecules. We considered the adhesive kinetics based on Bell's law of bond dissociation [7]. However, in several cases a more complex behavior has been observed [17].

For further studies it is instructive to consider the vWF and GP1b with the dynamics of catch- and slip-bonds observed in atomic force microscopy [16] and flow chamber experiments [17]. Another possible improvement of the model is to simulate the platelet motion under hydrodynamic flows, typical for flow chamber experiments and studies in vivo. In this case the interplay between hydrodynamic forces, cell-cell collisions and bonds kinetics may lead to complex dynamics of cells and their aggregates. Finally, since platelets have several adhesive receptors, activated by force or chemical agonists, it would be interesting to simulate temporal dynamics of adherent cells after their activation. In this case, the elasticity of platelets also undergoes dramatic changes followed by to the cytoskeleton restructuring.

This study was supported by Russian Foundation for Basic Research (RFBR grants 1902-00480-a , 19-31-70002-mol-a-mos). AVB also acknowledges the support from RUDN University (5-100 project).

\section{References}

[1] Jackson S.P., Nesbitt W.S. and Westein E., Journal of Thrombosis and Haemostasis 7(Suppl. 1), 17-20 (2009).

[2] Belyaev A.V., Dunster J.L., Gibbins J.M., et al., Phys Life Rev 26-27, 57 (2018).

[3] Michelson A.D., Platelets 3rd Edition (Academic Press, 2013).

[4] Haga et al., Annals of Biomedical Engineering 26, 268-277 (1998).

[5] Kulkarni S.,Dopheide S.M., Yap C.L., et al., J Clin Invest 105, 783 (2000).

[6] Moore N.W., Mulder D.J., Kuhl T.L., Langmuir 24, 1212 (2008).

[7] Bell G.I., Science 200(4342), 618 (1978).

[8] Hammer D.A., Apte S.M., Biophys J 63, 35 (1992).

[9] Sircar S., Bortz D. M., Math Biosci 245(2), 314 (2013).

[10] Rakshit S., Zhang Y., Manibog K., et al., PNAS 109(46), 18815 (2012).

[11] Arya M., Kolomeisky A.B., Romo G.M., et al., Biophys J 88, 4391 (2005).

[12] Israelachvili J., Min Y., Akbulut M., et al., Rep Prog Phys 73(3), 036601 (2010).

[13] Mody and King, Biophys J 95, 2539-2555 (2008).

[14] Asmolov E.S., Belyaev A.V., Vinogradova O.I., Phys Rev E 84, 026330 (2011).

[15] N.N. Kalitkin, P.V. Koryakin, Numerical methods, book 2 (2013) 304.

[16] Lining Ju et al., Thrombosis Research 136, 606-612 (2015).

[17] L.A.Coburn et al., Biophys J 100, 304-312 (2011). 